\title{
Book Review of Green Materials for Sustainable Water Remediation and Treatment
}

\author{
Nour-Eddine Es-Safi \\ Mohammed V Agdal University, Rabat, Morocco \\ Email: $\underline{\text { n.essafi@ens.um5a.ac.ma }}$
}

Received 15 June 2014; revised 13 July 2014; accepted 11 August 2014

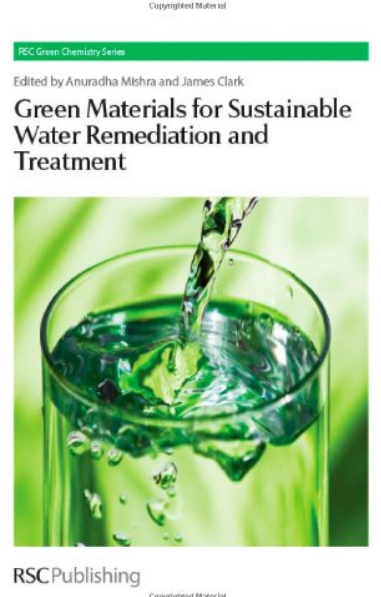

\author{
Green Materials for Sustainable \\ Water Remediation and Treatment \\ Edited by \\ Anuradha Mishra \\ and \\ James H. Clark
}

RSC 2013, 259 p

ISBN: 9781849736213

Water is one of the most important substances on earth. Safe drinking water is essential to humans and other life forms. All plants and animals must have water to survive. If there was no water, there would be no life on earth. Apart from drinking it to survive, people have many other uses for water. This natural resource is becoming scarcer and its demand exceeds supply in some region rendering its availability a major social and economic concern. Apart of this scarcity is water pollution which is increasing day by day in many parts of the world. Sources of fresh water on land are getting more and more polluted than ever before. As a result, contaminated water became unsuitable for use. Poor water quality is deadly since contaminated water causes hazards to public health through poisoning or the spread of disease. In order to address these issues, the practice of water remediation has been developed.

Water remediation is the process that is used to remove pollution from water. This is a type of environmental cleanup which focuses on addressing pollution of water supplies. The goal of a water remediation is to turn polluted water into clean water, or to sequester polluted water so that people will not be exposed to danger and to prevent the spread of the pollutant. Water remediation techniques include biological, chemical, and physical treatment technologies. The importance of water remediation gave recently rise to several studies focusing on different aspect of this field. 
In this context the book entitled Green Materials for Sustainable Water Remediation and Treatment has recently been published. The book is a collection of 11 interesting chapters dedicated to different aspects of water remediation. The different chapters are written by specialists from different universities. The chapters cover the fundamentals of water remediation techniques and the challenge of using them. The subjects are covered in depth and linked to up-dated references. The contributing authors are experts on the subjects and are well known in their area. The book chapters give essential information about several types of green materials used for water remediation which could allow a sustainable way of treating polluted water.

Chapter 1 gives and discusses the guidelines and the directives being followed for materials to be used for water remediation. Chapter 2 gives a generalized overview of the currently available green materials for sustainable remediation of metals in water including both biological and chemical methods. The role and the use of plant-biomass materials in heavy metal treatment of contaminated water are presented in Chapter 3. Various types of biomass for metal removing from waste water in addition to the mechanisms involved in the process are also presented and discussed.

Chapter 4 presents the flocculation technique and the use of flocculants for the treatment of wastewater. Plant and animal polysaccharides are thus presented as flocculants in effluent treatment. Chapter 5 presents an extensive overview on zeolites and their application in wastewater treatment. A presentation of water softening and applications for removal of ammonia from wastewater are also given in this chapter.

Chapter 6 presents functionalized silicagel as green material for metal remediation. Synthesis benefits and application of such material as chelating sorbents are discussed. Chapter 7 presents nanotechnology as one of the recently developed technology and its application in the field of water remediation. Various types of nanomaterials such as metal-containing nanoparticles, carbonaceous nanoparticles, nanocrystalline zeolites, photocatalyss, magnetic nanoparticles, and dendrimers are thus presented as potent materials for water remediation. Chapter 8 presents ionic liquids as new potent green solvents used in many extraction and separation processes. A brief review on different types of ionic liquids is thus presented. Their interesting properties were thus leveraged in water remediation and applied to metal extraction.

Chapter 9 describes periphyton biofilms and their potential as new materials for sustainability of aquatic ecosystems. After a brief presentation of the composition and structure of pyriphyton biofilm, the chapter describes their application of water remediation as water and wastewater purification. The use periphyton biofilms as inhibitors of phorphorous release from sediments and in the control of cyanobacterial bloom are also presented. Chapter 10 describes dyes as water polluters and the use of microorganisms for its remediation. The use of green viable algal biomass for the treatment of textiles wastewater is described. The mechanism involved and factors affecting biosorption and the parameters used for predicting the efficacy of the use of viable green algae are also discussed. Chapter 11 focuses on surfactants and their use as green materials for water remediation. The behavior of surfactants in aqueous systems is described. Factors such as specific surface area, surface charge/ion-exchange capacity, porosity and so on which affect surfactant modification of solid media for removal of oxo ions are described.

Overall the book presents a focused set of chapters covering a range of topics in the field of green materials for water remediation including the synthesis of new materials, modification of natural materials and use of clean technologies for water purification. The book is suitable for new and established researchers in the field and could be a useful source for teaching material. The use of clear, up-to-date protocols for procedures is a great feature, and the diversity of remediation techniques that are described allows practitioners to assess the merits of adopting alternate approaches. In summary, Green Materials for Sustainable Water Remediation and Treatment will be a very useful addition to any student, researcher, practitioner and laboratory involved in water remediation. 
Scientific Research Publishing (SCIRP) is one of the largest Open Access journal publishers. It is currently publishing more than 200 open access, online, peer-reviewed journals covering a wide range of academic disciplines. SCIRP serves the worldwide academic communities and contributes to the progress and application of science with its publication.

Other selected journals from SCIRP are listed as below. Submit your manuscript to us via either submit@scirp.org or Online Submission Portal.
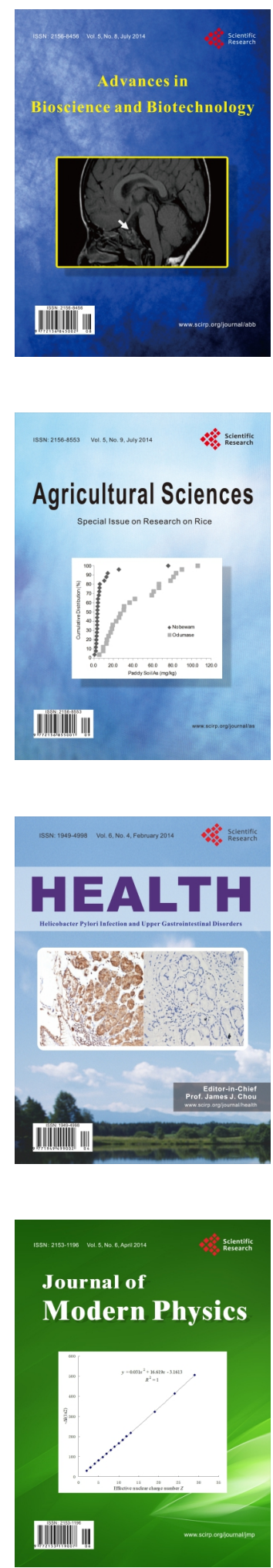
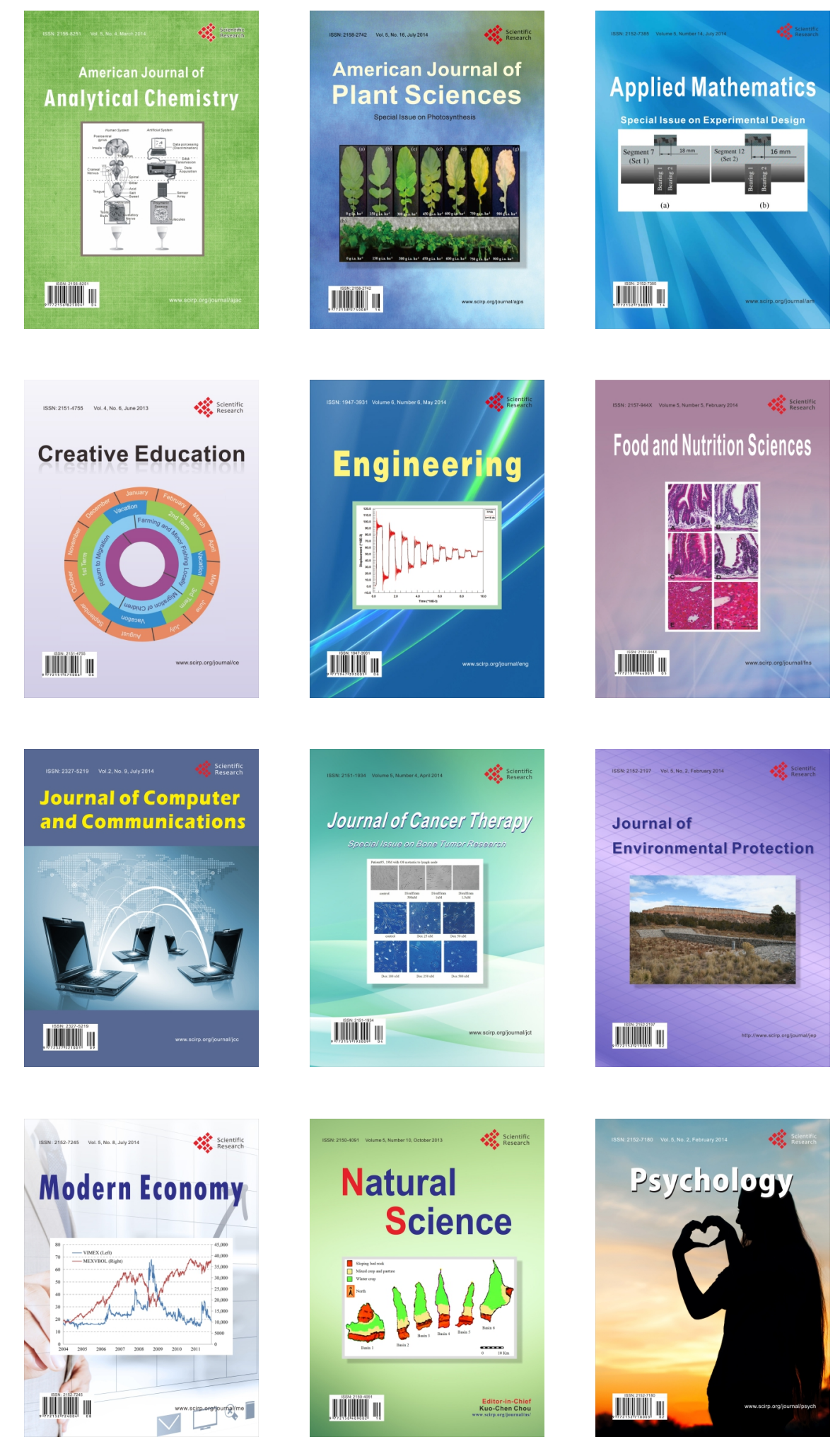